\title{
Relation longueur-poids et facteur de condition de sept espèces de poisson du réservoir de Samandeni avant son ouverture à la pêche (Burkina Faso).
}

\author{
Mahamoudou MINOUNGOU1, Raymond OUEDRAOGO², Nomwine DA², Adama OUEDA1. \\ 1: Laboratoire de Biologie et Écologie Animales, Unité de Formation et de Recherche en Sciences de la Vie et de la \\ Terre, Université Joseph KI-ZERBO, Burkina Faso. \\ 2.: Département Environnement et Forêts, Institut de l'Environnement et Recherches Agricoles, Burkina Faso. \\ *Auteur de la correspondance, Courriel : minoungou_mahamadou@yahoo.fr
}

Original submitted in on $11^{\text {th }}$ May 2020. Published online at www.m.elewa.org/journals/ on $31^{\text {st }}$ July 2020 https://doi.org/10.35759/JABs.151.5

\begin{abstract}
RESUME
Objectif : Fournir les premières données sur l'ichtyofaune du réservoir de Samandeni avant son ouverture à la pêche à travers l'étude de la relation longueur-poids et de facteur de condition de sept espèces dominantes dudit réservoir.

Méthodologie et résultats : Les poissons ont été collectés entre avril 2018 et janvier 2019 à travers des pêches expérimentales aux filets épervier et maillant. Sept espèces dominantes du réservoir à savoir Sarotherodon galilaeus (Mango tilapia), Coptodon zillii (Redbelly tilapia), Oreochromis niloticus (Tilapia du Nil), Marcusenius senegalensis (Trunkfish), Synodontis schall (Wahrindi), Schilbe intermedius (African butter catfish) et Brycinus nurse (Nurse tetra) ont été soumises à l'étude de la relation longueur-poids (RLP) et de facteur de condition. Les régressions longueur-poids, appliquées ici, ont donné des coefficients d'allométrie situés entre 2,86 et 3,20 avec des coefficients de détermination qui varient de 0,7312 à 0,9443.

Conclusion et application des résultats: Ces résultats qui sont d'une grande importance dans la modélisation de l'écosystème du réservoir de Samandeni, décrivent une croissance isométrique pour les sept espèces étudiées. En référence aux facteurs de condition, Sarotherodon galilaeus, Coptodon zillii, Oreochromis niloticus et Brycinus nurse présentent de bon état de forme.
\end{abstract}

Mots clés : ichtyofaune, relation longueur-poids, facteur de condition, réservoir de Samandeni, Burkina Faso.

Weight-length relationships and condition factor of seven species of fish from Samandeni reservoir before it was opened to fishing (Burkina Faso).

\footnotetext{
ABSTRACT

Objective: To provide the first data of the ichthyofauna of the Samandeni reservoir before it is opened to fishing through the study of the length-weight relationship and condition factor of seven dominant species of the said reservoir.

Methodology and Results: The fish were collected between April 2018 and January 2019 through experimental fishing with hawk and gillnets. Seven dominant species of the reservoir, namely Sarotherodon
} 
galilaeus (Mango tilapia), Coptodon zillii (Redbelly tilapia), Oreochromis niloticus (Nile Tilapia), Marcusenius senegalensis (Trunkfish), Synodontis schall (Wahrindi), Schilbe intermedius (African butter catfish) et Brycinus nurse (Nurse tetra) were subjected to the study of the length-weight relationship and condition factor. The length-weight regressions applied here gave allometric coefficients between 2.86 and 3.20 with coefficients of determination varying from 0.7312 to 0.9443 .

Conclusion and Application of results: These results, which are of great importance in the modelling of the ecosystem of the Samandeni reservoir, describe isometric growth for the seven species studied. With reference to the condition factors, Sarotherodon galilaeus, Coptodon zillii, Oreochromis niloticus and Brycinus nurse are in good shape.

Keywords: ichtyofauna, weight-length relationships, condition factor, Samandeni reservoir, Burkina Faso.

\section{INTRODUCTION}

L'étude des relations longueur-poids (RLP) a plusieurs applications en biologie de la pêche (Moutopoulos et Stergiou, 2002). Elle permet de déterminer le poids des poissons dont on connaît la longueur ou inversement et de décrire la forme, l'embonpoint des poissons et ses variations au cours de sa croissance (Sidibé, 2003). Ces données qui peuvent varier en fonctions de multiples facteurs tels que le sexe, le stade de maturité sexuelle, les conditions alimentaires, la pression due à la pêche (Khallaf et al., 2003), sont utilisées dans la modélisation des écosystèmes aquatiques ainsi que l'évaluation de leur rendement (Kulbicki et al., 2005). Selon Lorenzen (2000), les paramètres de la RLP fournissent des informations sur l'habitat des poissons et sont par ailleurs des outils de comparaison entre diverses populations d'une même espèce vivant dans des écosystèmes similaires ou différents (Stergiou et Moutopoulos, 2001 ; Thomas et al., 2003 ; Odat, 2003). Au Burkina Faso, les réservoirs d'eau artificiels sont les principaux supports de la production piscicole (Villanueva et al., 2006 ; Oueda et al., 2008). De toutes les études réalisées au Burkina Faso sur la biologie, aucune n'a été consacrée à la faune piscicole de ces réservoirs d'eau artificiels avant leur ouverture à la pêche. Les résultats de telles études sont pourtant nécessaires à la formulation du plan d'aménagement et de gestion de la pêcherie comme requis par la stratégie nationale halieutique et le code forestier du Burkina Faso de 2011 (Assemblée Nationale, 2011 ; Présidence du Faso, 2014). Seules quelques espèces des réservoirs du Sourou, de Boalin, et de la Kompienga ont respectivement été étudiées par Coulibaly
(2008), Ouedraogo et al. (2015) et Da et al. (2018) après plusieurs années d'exploitation voire de surexploitation (Mano, 2016). Ces données initiales, essentielles à l'élaboration d'une stratégie d'exploitation raisonnée des populations de poissons ont toujours fait défaut au Burkina Faso. Cette étude vient en réponse à ce problème d'absence de données initiales pour ce qui est du réservoir de Samandeni, nouvellement construit et mis en eau en mai 2017, et qui est par ailleurs le troisième plus grand réservoir du Burkina Faso. Des études de la RLP et de facteur de condition ont été conduites sur quelques espèces sur plusieurs retenues d'eau douce en Afrique. Ces études ont présenté des intérêts pratiques dans la gestion des stocks piscicoles où il importait, pour obtenir un rendement maximum, de disposer des paramètres aisément calculables et ayant des interprétations aussi bien biologiques qu'écologiques (Fafioye et Oluajo, 2005; Abobi et al., 2013; Laurence et al., 2019). Au Burkina Faso, cette approche est récente avec le Projet de gestion de la pêche dans le SudOuest du Burkina Faso (GPSO, 1995). La seconde étude du genre est celle de Coulibaly (2008) qui a permis de fournir des données de référence sur quatre espèces de poissons d'intérêt commercial dans la rivière Sourou. Par la suite, cette approche a permis à Sirima et al. (2009) de montrer une bonne croissance et une bonne adaptation des principales espèces d'intérêt commercial des cours d'eau de la partie Burkinabè du Bassin de la Comoé. Elle a également permis à Ouedraogo et al. (2015) d'apprécier l'état de forme physiologique de trois espèces de la famille des Cichlidae du lac sahélien de Higa, un site Ramsar du Burkina Faso. 
La dernière étude en date est celle de Da et al. (2018), qui à travers l'étude comparée des RLP et l'état de " bien-être » de Clarias anguillaris et de Sarotherodon galilaeus, a montré le niveau avancé de dégradation de l'écosystème du lac Bam par rapport au réservoir de la Kompienga au Burkina Faso. Dans le contexte actuel du Burkina Faso, marqué par une demande de plus en plus forte en poisson estimée à 130000 tonnes par an pour une production halieutique nationale estimée à 24000

\section{MATERIEL ET METHODES}

Site d'étude : Le réservoir de Samandeni est localisé au sud-ouest du Burkina Faso, dans le bassin supérieur du Mouhoun et près du village Samandemi. La digue du réservoir de Samandeni est à environs $50 \mathrm{~km}$ de BoboDioulasso. Le réservoir de Samandemi s'étend sur cinq communes rurales dont trois de la province du Houet (Bama, Banzon et Karangasso-Sambla), et deux de la province du Kénédougou (Samorogouan, Kourouma). Nouvellement construit et mis en eau en mai 2017, ce réservoir fait suite à une politique de construction de retenues d'eau artificielles entamée depuis les indépendances du Burkina Faso afin de remédier à la rareté de ses eaux de surface, comme dans la plupart des pays sahéliens de l'hinterland (Piquemal, 1991 ; Poda et al., 2004). Le réservoir de Samandeni a une capacité de stockage de 15 milliards de mètres cubes tonnes en 2018, avec un niveau moyen de consommation estimé à $6,6 \mathrm{~kg} / \mathrm{an} / \mathrm{hab}$. (MRAH 2018), il est apparu essentiel de mener cette étude de la RLP et de facteur de condition des espèces dominantes du réservoir de Samandeni avant son ouverture à la pêche afin de mettre à la disposition des décideurs, des données susceptibles d'aider à l'élaboration d'une politique de gestion optimale et durable dudit réservoir.

d'eau et permettra à termes la création d'une zone agroindustrielle ainsi que l'aménagement de 21000 hectares de périmètres en maitrise totale d'eau repartis sur 17 sites (Attakin, 2006). Avec ces 15000 ha de superficie inondée, le réservoir de Samandeni est la troisième plus grande étendue d'eau artificielle du pays après Bagré (25000 ha) et Kompienga (20000 ha) (Yonkeu et al. 2009). Cinq stations d'échantillonnage ont été choisies le long du réservoir en aval de la digue. II s'agit des stations de Banzon, Bado-ville, Dioufoulma, Sadina et Sikorola. La figure 1 présente la position géographique du réservoir au sein du bassin du Mouhoun sur la carte du Burkina Faso et les cinq stations d'échantillonnage. Le tableau 1 décrit les berges des stations d'échantillonnage, la distance qui sépare chacune d'elle de la digue ainsi que leurs coordonnées GPS. 


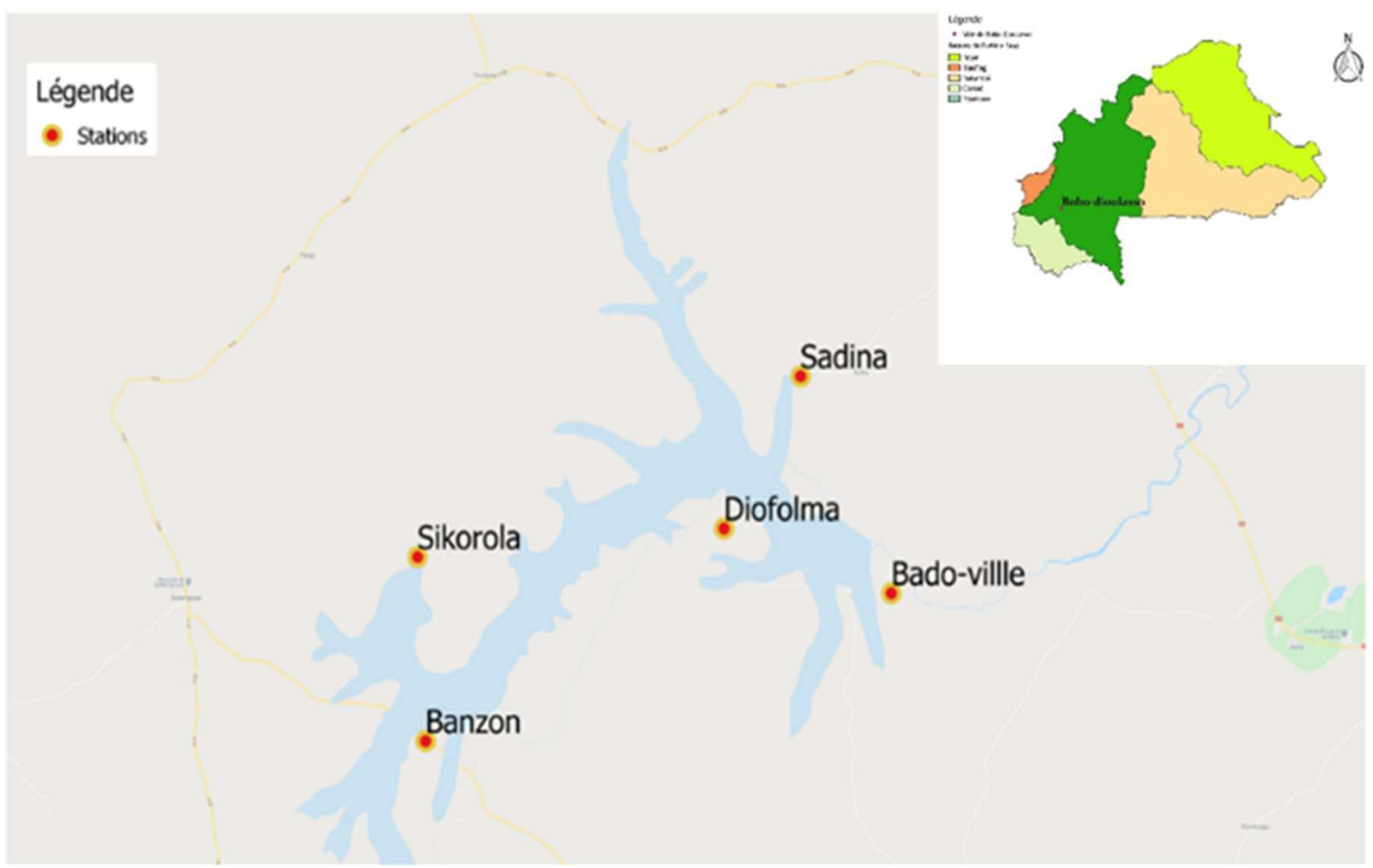

Figure 1 : La position géographique du réservoir de Samandeni et des stations d'échantillonnage

Tableau 1 : Quelques caractéristiques des cinq stations d'échantillonnage

\begin{tabular}{|c|c|c|c|c|}
\hline Station & $\begin{array}{l}\text { Longitude } \\
\text { (N) }\end{array}$ & Latitude (W) & $\begin{array}{l}\text { Distance } \\
\text { Station - } \\
\text { Digue }\end{array}$ & Nature des berges \\
\hline Bado-ville & $11^{\circ} 23^{\prime} 55,90^{\prime \prime}$ & $004^{\circ} 34^{\prime} 41,40^{\prime \prime}$ & $00 \mathrm{Km}$ & $\begin{array}{ll}- & \text { pas de végétation, } \\
- & \text { présence d'activités anthropiques (la } \\
\text { digue du barrage), } \\
-\quad \text { présence d'hippopotames }\end{array}$ \\
\hline Banzon & $11^{\circ} 19^{\prime} 59,83^{\prime \prime}$ & $004^{\circ} 46^{\prime} 00,14^{\prime \prime}$ & $24 \mathrm{Km}$ & $\begin{array}{ll}- & \text { forte activités anthropiques (riziculture), } \\
- & \text { construction d'une digue de } \\
\text { soutènement du village, } \\
\text { - } \quad \text { présence d'hippopotames. }\end{array}$ \\
\hline Dioufoulma & $11^{\circ} 23^{\prime} 00,28^{\prime \prime}$ & $004^{\circ} 37^{\prime} 32,41^{\prime \prime}$ & $09 \mathrm{Km}$ & $\begin{array}{ll}- & \text { village nouvellement délocalisé, } \\
\text { - } & \text { pas d'activités anthropiques, } \\
- & \text { présence de troncs d'arbres morts, } \\
\text { - } & \text { présence d'hippopotames. }\end{array}$ \\
\hline Sadina & $11^{\circ} 26^{\prime} 53,22^{\prime \prime}$ & $004^{\circ} 37^{\prime} 43,20^{\prime \prime}$ & $11 \mathrm{Km}$ & $\begin{array}{ll}- & \text { village non encore délocalisé, } \\
- & \text { zone forestière, } \\
- & \text { présence d'activités anthropiques } \\
\text { (culture de mil, élevage), } \\
-\quad \quad \text { présence d'hippopotames }\end{array}$ \\
\hline Sikorola & $11^{\circ} 25^{\prime} 24,55^{\prime \prime}$ & $004^{\circ} 43^{\prime} 19,22^{\prime \prime}$ & $24 \mathrm{Km}$ & $\begin{array}{ll}- & \text { village partiellement délocalisé, } \\
- & \text { zone forestière, } \\
- & \text { forte activités anthropiques (bananeraie), } \\
- & \text { présence d'hippopotames }\end{array}$ \\
\hline
\end{tabular}


Méthodologie : Les poissons ont été échantillonnés à la suite de pêches expérimentales au moyen de filet épervier de 15 millimètres côté de maille et de filets maillants de $20 ; 30 ; 45 ; 60$ et 65 millimètres de côté de maille. Pour la pêche aux filets maillants, les engins ont été posés entre $16 \mathrm{~h}$ et $17 \mathrm{~h}$ et relevés le lendemain entre $08 \mathrm{~h}$ et $09 \mathrm{~h}$. Pour la pêche au filet épervier, au moins 50 jets de filet ont été effectués par site à chaque sortie. Deux pêcheurs professionnels ont été recrutés pour conduire ces pêches expérimentales qui ont été réalisées à pieds et/ou en pirogue en fonction de la profondeur de la zone prospectée. L'échantillonnage s'est étalé sur 10 mois entre avril 2018 et janvier 2019 avec quatre campagnes d'échantillonnages, respectivement en avril, juillet, novembre 2018 et janvier 2019. Les poissons ont été identifiés grâce à la clé de Paugy et al. (2003). Notons qu'en raison de l'interdiction

\section{RESULTATS}

Les espèces dominantes du réservoir de Samandeni sont : Sarotherodon galilaeus (Linnaeus, 1758), Coptodon zillii (Gervais, 1848), Oreochromis niloticus (Linnaeus, 1758), Marcusenius senegalensis (Steindachner, 1870), Synodontis schall (Bloch \& Schneider, 1801), Schilbe intermedius (Rüppell, 1832) et Brycinus nurse (Rüppell, 1832). Ces sept espèces, considérées dominantes, représentent $61,95 \%$ en termes d'abondance. Le Tableau 2 donne les caractéristiques des spécimens étudiés ainsi que les paramètres des équations de croissance des sept espèces. Les longueurs totales des spécimens varient de $1,50 \mathrm{~cm}$ chez Coptodon zillii à $35,50 \mathrm{~cm}$ chez Synodontis schall avec des poids qui varient entre 41,27 $\mathrm{g}$ chez Brycinus nurse et $129,49 \mathrm{~g}$ chez Synodontis schall. Les données de longueur et de poids sont récapitulées dans le Tableau 2. Les corrélations entre la longueur et le poids des spécimens sont très fortes avec de la pêche durant la période de l'étude, nous n'avons pas observé de pêche commerciale. Des espèces rencontrées, seules celles ayant des abondances relatives supérieures à $4 \%$, ayant été rencontrées dans au moins trois des cinq stations et pendant toute la période de collecte, ont été prises en compte pour cette étude.

Partant de l'équation de Le Cren (1951), $P=a * L^{b}$ où $\mathrm{L}$, est la longueur totale du poisson en $\mathrm{cm}$ et $\mathrm{P}$, le poids total du poisson en $\mathrm{g}$, les paramètres $a$ et $b$, représentant respectivement l'intercepte et le coefficient d'allométrie ont été déduits par linéarisation logarithmique.

Le Facteur de condition de Fulton $(K)$ a été calculé afin d'apprécier l'état d'embonpoint des poissons, suivant la formule : $K=100 *(P) /(L)^{b}$ (Bagenal et Tesch, 1978).

des coefficients de détermination qui vont de 0,7312 à 0,9443 . Quatre des sept espèces étudiées à savoir Schilbe intermedius, Sarotherodon galileaus, Marcusemius senegalensis et Synodontis schall ont une allométrie positive contrairement aux trois autres espèces (Coptodon zillii, Oreochromis niloticus et Brucinus nurse). Quant aux facteurs de condition K, les résultats au Tableau 2, nous indiquent que les trois espèces de la famille des Cichlidae ainsi que Brycinus nurse présentent un embonpoint contrairement à Schilbe intermedius, Marcusenius senegalensis et Synodontis schall. L'analyse des facteurs de condition K en fonction des saisons indique une faible variation des valeurs selon les saisons. Néanmoins, Sarotherodon galilaeus, Schilbe intermedius, Marcusenius senegalensis et Synodontis schall ont des K légèrement supérieurs en saison sèche qu'en saison pluvieuse (Tableau 3). 
Tableau 2 : Paramètres de la relation poids-longueur et facteur de condition $\mathrm{K}$ des sept espèces.

\begin{tabular}{|c|c|c|c|c|c|c|c|c|c|c|c|c|c|}
\hline \multirow[b]{2}{*}{ Espèces } & \multirow{2}{*}{$\mathbf{N}$} & \multicolumn{3}{|c|}{ Longueur $(\mathrm{cm})$} & \multicolumn{3}{|c|}{ Poids (g) } & \multirow{2}{*}{ a } & \multirow{2}{*}{ b } & \multirow{2}{*}{$\mathrm{Cr}$} & \multirow{2}{*}{$\mathbf{R}^{2}$} & \multicolumn{2}{|c|}{$\mathrm{K}$} \\
\hline & & Min & Moy & Max & Min & Moy & $\operatorname{Max}$ & & & & & Moy & Ec \\
\hline Sarotherodon galilaeus & 590 & 4,00 & 17,71 & 28,70 & 0,5 & 119,88 & 421,6 & 0,013 & 3,15 & $A+$ & 0,92 & 1,19 & 0,2699 \\
\hline Schilbe intermedius & 331 & 6,1 & 17,92 & 31,20 & 1,00 & 44,50 & 264,20 & 0,015 & 3,20 & $A+$ & 0,94 & 0,37 & 0,0726 \\
\hline Coptodon zillii & 229 & 1,5 & 15,25 & 23,60 & 0.076 & 82,62 & 256,80 & 0,022 & 2,91 & $A-$ & 0,92 & 2,53 & 2,1310 \\
\hline Marcusenius senegalensis & 196 & 11,2 & 15,61 & 29,60 & 13,70 & 85,50 & 547,00 & 0,005 & 3,12 & $A+$ & 0.73 & 0,66 & 0,2952 \\
\hline Oreochromis niloticus & 195 & 1,80 & 15,61 & 26,70 & 6,90 & 85,50 & 240,19 & 0,103 & 2,86 & $A-$ & 0,94 & 11,73 & 2,6415 \\
\hline Synodontis schall & 171 & 13,40 & 22,92 & 35,50 & 30,90 & 129,49 & 591,00 & 0,007 & 3,11 & $A+$ & 0,85 & 0,69 & 0,1474 \\
\hline Brycinus nurse & 140 & 6,5 & 15,74 & 26,70 & 1 & 41,27 & 160,50 & 0,012 & 2,95 & A- & 0,83 & 1,14 & 0,1739 \\
\hline
\end{tabular}

$\mathrm{N}=$ Nombre de spécimens, $C r=t y p e$ de croissance, $A+=$ allométrie positive, $A=$ allométrie négative, $K=F a c t e u r$ de condition, Moy=moyenne et Ec=Ecart-type,

Min=minimum, Max=maximum

Tableau 3: Facteur de condition selon les saisons des sept espèces

\begin{tabular}{l|c|c}
\hline \multirow{2}{*}{ Espèces } & \\
\cline { 2 - 3 } & Saison sèche & Saison pluvieuse \\
\hline Sarotherodon galilaeus & 1,288 & 0,985 \\
Schilbe intermedius & 0,381 & 0,372 \\
Coptodon zillii & 2,528 & 2,547 \\
Marcusenius senegalensis & 0,676 & 0,601 \\
Oreochromis niloticus & 11,566 & 12,068 \\
Synodontis schall & 0,712 & 0,686 \\
Brycinus nurse & 1,133 & 1,139 \\
\hline
\end{tabular}




\section{DISCUSSION}

Les résultats donnent pour Sarotherodon galilaeus, une valeur du coefficient d'allométrie $b$ supérieure à celles obtenues par Da et al. (2018) pour la même espèce, dans le lac Bam $(b=2,41)$ et dans le réservoir de la Kompienga $(b=3,08)$ au Burkina Faso, deux écosystèmes soumis à différents niveaux de pressions environnementales et anthropiques. La valeur de $b$ de $S$. galilaeus est également supérieure à celle obtenue par Montchowui et al. (2009) dans le lac Hlan au Bénin ( $b=$ 2.850) soumis à une exploitation humaine relativement faible en raison de mesures réglementant la pêche traditionnelle. Selon les conclusions de ces études, la valeur de $b$ semble faible dans les écosystèmes dégradés par une surexploitation des ressources piscicoles. Cette assertion est en phase avec les résultats de cette étude et pourrait s'expliquée par la récente mise en eau du réservoir de Samandeni (mai 2017) doublée de l'interdiction de la pêche pendant la période de l'étude. Aussi, une valeur de $b$ supérieure à 3 dénote que $S$. galilaeus prend de l'embonpoint au cours de sa croissance en longueur. Selon Lauzanne (1988), la création de barrage engendre dès les premiers moments, une prolifération excessive de microphytes et de macrophytes compris dans la gamme de nourritures de prédilection des Cichlidae (Njiru et al., 2004). Ainsi, ces résultats suggèrent que $S$. galilaeus a un bien meilleur état de "bien-être » et une bonne croissance au sein du réservoir de Samandeni soumis à un faible degré d'exploitation au cours de la période de l'étude. Nehemia, (2012) à travers une étude expérimentale a montré que Coptodon zillii est une espèce qui s'adapte mieux en eau douce que dans des étangs d'eau de mer avec des valeurs de $b$ respectivement égales à 2,94 et 2,07 . La valeur du coefficient d'allométrie $(b=2,91)$ obtenue lors de cette étude est située entre ces deux valeurs expérimentales de Nehemia (2012), mais se rapproche davantage de celle obtenue en eau douce. La valeur de $b$ inférieure à celle obtenue expérimentalement en eau douce par Nehemia (2012) pourrait s'expliquer par le fait qu'en milieu expérimental, dénuées de prédation et de compétitions entre les espèces sympatriques, les conditions de vie des poissons sont généralement optimales par rapport à leurs conditions de vie naturelle (Legendre et Ecoutin, 1996). Le coefficient d'allométrie de Oreochromis niloticus obtenue dans cette étude, suggère que cette espèce a une croissance de type allométrique négative. Contrairement à ce résultat, Olurin et Aderibigbe (2002) ont rapporté sur 0 . niloticus dans une ferme piscicole de Sanni Luba ljebu-Ode, dans l'État d'Ogun au Nigeria, une forme de croissance isométrique $(b=3,10)$. Ces résultats sont conformes à ceux de Fagade (1983) sur un Cichlidae apparenté, Chromidotilapia guntheri du lac artificiel d'Ibadan, au sud-ouest du Nigéria. De telles approches comparatives entre espèces sympatriques d'un même écosystème nous permettent d'en faire autant pour les Cichlidae du réservoir de Samandeni. Ainsi, les résultats suggèrent que 0 . niloticus est dans un état de "bien-être » physiologique moins meilleur que $S$. galilaeus et $C$. zillii pendant la période de l'étude. Omnivore-brouteur, 0 . niloticus est une espèce qui se nourrit essentiellement de phytoplancton, de périphyton et de détritus (Lauzanne, 1988). O. niloticus est également connue pour sa préférence des eaux peu profondes avec une activité essentiellement diurne. D'où sa présence auprès des berges (Mukankomeje et al., 1994). Au cours des travaux d'échantillonnage, nonobstant l'interdiction de la pêche, des pièges à poissons tels que des nasses, des filets maillants et des preuves de pêche clandestine des espèces facilement accessibles à partir des berges, ont été observés le long du réservoir de Samandeni. La faible valeur du coefficient d'allométrie de 0 niloticus pourrait être due aux perturbations liées au braconnage des espèces vivant au bord des berges du réservoir comme 0 niloticus. Selon les résultats du projet de Gestion de la Pêche dans le Sud-Ouest du Burkina Faso (GPSO, 1995) Brycinus nurse a une croissance allométrique positive avec un $b$ de 3,53 . Cette valeur de $b$ tout comme celles obtenues par Abobi et al., (2013) dans les cours inférieurs de la Volta Blanche à Yapei au Ghana $(b=$ 3,0737 ) et par Konan et al., (2007) dans les rivières du Sud-Est de la Côte d'Ivoire où des nutriments abondent, sont supérieures à celle obtenue à Samandeni $(b=$ 2,95). La faible valeur de $b$ de $B$. nurse obtenue dans cette étude pourrait une fois de plus s'expliquer par la récente mise en eau du réservoir de Samandeni, pourvu de ressources alimentaires nouvelles, certes extrêmement abondantes mais peu variées. $B$. nurse essentiellement insectivore et par moment détritivore (Ouéda et al., 2008) se trouve dans ces circonstances face à une faiblesse des ressources en insectes mais également en compétition avec les espèces essentiellement détritivores. Cela pourrait expliquer la faible valeur de $b$ pour $B$. nurse. En revanche, la valeur de $b$ de $B$. nurse de cette étude, est supérieure à celle obtenue par de Oni et al. (1983) dans la rivière surexploitée de Galma au Nigeria et qui est de 2,668. La RLP de Schilbe intermedius de cette étude montre une valeur de $b(2,95)$ inférieure à celles obtenues par Abobi 
et al. (2013) dans les cours inférieurs de la Volta Blanche à Yapei au Ghana $(b=3,4592)$ et par Ecoutin et al., (2005) dans les eaux d'un estuaire non perturbé de la Gambie qui est de 3,259. II en est de même avec Konan et al. (2007) dans les rivières du Sud-Est de la Côte d'Ivoire où $b$ est de 3,360. De Vos (1995) note que $S$. intermedius est un prédateur qui se nourrit en surface et de nuit. Le braconnage effectué de nuit par les pêcheurs clandestins peut donc perturber sa prise de nourriture et pourrait expliquer l'allométrie négative observée pendant l'étude. La valeur de $b$ de Marcusenius senegalensis de cette étude suggère une croissance allométrique positive. Adjibade et al. (2019) ont trouvé un résultat pareil chez certains Mormyridae dont $M$. senegalensus dans le fleuve Niger au nord du Bénin. Cependant, Akintade et al. (2016) ont obtenu dans le ruisseau de Badagry à Lagos au Nigéria, une valeur de 2,12. II en est de même des résultats du projet de Gestion de la Pêche dans le Sud-Ouest (GPSO, 1995) qui a trouvé une valeur de $b$ de 2,86 pour $M$. senegalensis. Cette croissance allométrique positive suggère que $M$. senegalensis de Samendeni prend plus de poids à mesure que sa longueur augmente. II est donc probable que cette espèce montre une tolérance relativement élevée aux conditions environnementales de Samendeni pendant la période de l'étude. Globalement, Sarotherodon galilaeus, Schilbe intermedius, Marcusenius senegalensis et Synodontis schall ont des valeurs de $b$ supérieures à 3 . Ce qui suggère que pour ces espèces, la croissance est de type allométrique positive contrairement aux trois autres espèces. Pour les sept espèces étudiées, la valeur moyenne d'allométrie $b(3,043 \pm 0,133)$ indique que la « loi cubique » (Froese, 2006) pourrait être applicable pour les poissons de Samandeni car les valeurs de $b$ varient de 2,86 à 3,20 . Cette fourchette de la valeur de $b$ est conforme aux valeurs typiques des espèces ayant une croissance isométrique $(b=3)$ habituellement rapportée par la littérature et qui se situent entre 2,50 et 3,50 (Calander, 1969 ; Pauly et Moreau, 1997 ; Froese, 2006). En effet, la pente de la droite de régression $b$ exprime la forme relative du corps d'un poisson (Le Cren, 1951 ; Kareem et al., 2015 ; Froese et al., 2014) et peut être influencées par le sexe, la phase de croissance, les contenus stomacaux, le niveau du développement des gonades (Hossain et al., 2006) et les conditions de l'environnement (Baby et al., 2011).
Certains auteurs tels que Lalèyè (2006); Muzzalifah et al. (2015); Lederou et al. (2016), sont arrivés aux mêmes conclusions quand bien même ces paramètres n'ont pas été pris en compte spécifiquement lors des traitements des données. Alhassan et al. (2015) considère qu'un coefficient $K$ inférieur à 1 suggère que les poissons sont dans de mauvaises conditions et un coefficient supérieur à 1 suppose le contraire. Dès lors, l'on peut admettre que les trois espèces de la famille des Cichlidae à savoir Sarotherodon galilaeus, Coptodon zillii, et Oreochromis niloticus ainsi que Brycinus nurse étaient au moment de l'étude, en bonnes conditions. $\mathrm{Ce}$ qui n'était pas le cas chez Schilbe intermedius, Marcusenius senegalensis et Synodontis schall. Cet état de méforme de ces trois espèces l'est davantage en saison pluvieuse au regard des valeurs de leurs $\mathrm{K}$ respectifs, encore plus faible en saison pluvieuse par rapport à celles obtenues en saison sèche. Selon des auteurs tels que Lowe-Mc Connell (1966); Obeng (1969) ; Lawson et al. (1969) ; Lelek et Elzarka (1973); Balon et Coche (1974); Lewis (1974) et Lausanne (1988), la création de lac de barrage comme celui de Samandeni pose de grands changements dans l'éventail des ressources alimentaires présentes dans les cours d'eau avant la création des réservoirs et leur mise en eau. Et effet, ces auteurs soutiennent que la faune d'invertébrés proprement benthiques subit dans un premier temps une décroissance spectaculaire due sans doute aux mauvaises conditions d'oxygénation dès les premiers moments de la mise en eau. Devant ces ressources alimentaires nouvelles, peu variées, mais extrêmement abondantes, les poissons, notamment ceux faisant partie des consommateurs tertiaires telles que Schilbe intermedius et Synodontis shall vont devoir s'adapter (Lausanne, 1988). Ce qui expliquerait les faibles valeurs de $\mathrm{K}$ de ces deux espèces et encore plus en saison pluvieuse. Ce phénomène serait d'ailleurs la cause de la disparition de certaines espèces benthiques comme les Mormyridae (Lausanne, 1988). C'est le cas de Marcusenius senegalensis, dans le réservoir de Samandeni dont la valeur de k est 0,66. En somme, la récente mise en eau du réservoir de Samandeni, intervenue seulement en mai 2017, pourrait expliquer l'état de "bien-être » physiologique plus ou moins bonne des sept espèces de poissons soumises à cette étude. 


\section{CONCLUSION}

Les résultats combinés de la RLP et des facteurs de condition $\mathrm{K}$ nous font dire que globalement, les sept espèces étudiées présentent une bonne adaptation au sein du réservoir de Samandeni nouvellement mis en eau. Ces premières données qui sont d'une grande utilité dans la gestion rationnelle des stocks piscicoles du réservoir, le seront davantage si la même étude est répétée les deux premières années après l'ouverture de la pêche afin non seulement de poursuivre l'inventaire des espèces piscicoles mais également et surtout d'apprécier l'état de "bien-être » des poissons du réservoir dans le temps.

CONFLITS D'INTERET : Les auteurs déclarent qu'il n'y a aucun conflit d'intérêt.

\section{REMERCIEMENTS}

Nous remercions le projet Susfish plus et la Direction Générale des Ressources Halieutiques du Burkina Faso

\section{BIBLIOGRAPHIE}

Abobi SM, Ekau W. 2013. Length-Weight Relationships and Condition Factors of Alestes Baremoze, Brycinus Nurse and Schilbe Intermedius from the Lower Reaches of White Volta River (Yapei), Ghana. International Journal of Fisheries and Aquaculture. 5(6) : 152-165.

Akintade AO, Edwin CO, Simon EA. 2016. Lengthweight relationship, condition factor and sexratio of fish fauna in Badagry Creek, Lagos, Nigeria. International Journal of Marine Science, 6.

Alhassan EH, Akongyuure DN, Asumang F. 2015. Determination of Morphometric Relationship and Condition Factors of Four Cichlids from Golinga Reservoir in Northern Region of Ghana. Online Journal of Biological Science, 15(3): 201-206. DOI: http://dx.doi.org/10.3844/ojbsci.2015.20 1.206.

Assemblée Nationale. 2011: Loi N003-2011/AN portant Code Forestier au Burkina Faso.

Attakin E. 2006. Avant-projet détaillé du barrage de Samendeni. Etude d'impact sur l'environnement et le social. Mémoire de fin de formation d'études supérieures spécialisées. 2iE Groupe EIER/ETSHER, Burkina Faso.

Baby F, Tharian J, Abraham KM, Ramprasanth MR, Ali A, Ranghavan R. 2011. Length-weight relationship and condition factor of an endemic stone sucker, Garra gotyla stenorhynchus (Jerdon, 1849) from two opposite flowing rivers in southern Western Ghats. Journal of threatened taxa 3(6): 1851-1855.

Bagenal TB, Tesch AT. 1978. Conditions and Growth Patterns in Fresh Water Habitats. Blackwell Scientific Publications, Oxford, 75-89. pour l'assistance financière et logistique reçue lors de la collecte des données sur le site.

Balon EK. and Coche AG. 1974. Lake Kariba. A manmade tropical ecosystem in Central Africa. $\mathrm{Dr}$ W. Junk B.V. Publishers, The Hague, 767 p.

Calander KD. 1969. Handbook of Freshwater Fishery Biology. The lowa State Univ. Press, Ames, IA Lowa State 1: 752 pp.

Coulibaly ND. 2008. Relation longueur-poids chez quatre espèces de poissons de la rivière Sourou au Burkina Faso. Int. J. Biol. Chem. Sci., 2(3), pp. 331-338. http://dx.doi.org/10.4314/ijbcs.v2i3.3974 4.

Da N, Ouedraogo R, Oueda A. 2018. Relation poidslongueur et facteur de condition de Clarias anguillaris et Sarotherodon galilaeus pêchées dans le lac Bam et le réservoir de la Kompienga au Burkina Faso. Int. J. Biol. Chem. Sci. 12(4) : 1601-1610. ISSN 1997-342X (Online), ISSN 1991-8631

(Print).

http://ajol.info/index.php/ijbcs, http://indexmedicus.afro.who.int.

De Vos L. 1995. A systematic revision of the African Schilbeidae (Teleostei: Siluriformes). Ann. Mus. R. Afr. Centr., 271 (414 pp).

Ecoutin JM, Albaret JJ, Trape S. 2005. Length-weight relationships for fish populations of a relatively undisturbed tropical estuary: the Gambia. Fisheries Research, 72 (2-3), 347351.

Fagade SO. 1983. The biology of Chromidotilapia guntheri from a small lake. Arch Hydrobiol., 97: $60-72$

Froese R. 2006. Cube law, condition factor and weightlength relationships: history, meta-analysis, and recommendations. Journal of Applied Ichthyology, 22 (4): 241-253. DOI: 
http://dx.doi.org $\quad$ /10.1111/j.14390426.2006.00805. $x$.

Froese R, Thorson JT, and Reyes Jr. RB. 2014. A Bayesian approach for estimating lengthweight relationships in fishes. Journal of Applied Ichthyology, 30(1), 78-85.

Gayanilo Jr FC, Sparre P, Pauly D, Center W. 2005. FAO-ICLARM Outils d'évaluation des stocks II (FiSAT II). Version révisée. Guide d'utilisation.

GPSO (Projet Gestion des Pêches dans le Sud-Ouest). 1995. Enquêtes Halieutiques sur les Six Retenues : Tounoura, Comoé, Douna, Tiéfora, Lemouroudougou et Navrikpê. Situation et Recommandations. GOPA-COFAD: Bad Homburg.

Hossain MY, Ahmed ZF, Leunda PM, Jasmine S, Oscoz J, Miranda R, Ohtomi J. 2006. Condition, length-weight and length-length relationships of the Asian striped catfish Mystus vittatus (Bloch, 1794) (Siluriformes: Bagridae) in the Mathabhanga River, Southwestern Bangladesh. Journal of Applied Ichthyology 22 . 304-307.

Kareem OK, Olanrewaju AN, Orisasona O. 2015. Length-weight Relationship and Condition factor of Chrysichythys nigrodigitatus and Schilbe mystus in Erelu Lake, Oyo State, Nigeria. Journal of Fisheries and Livestock Production 3 (4): 1-4.

Khallaf E, Galal M, Athuman M. 2003. The biology of Oreochromis niloticus in a polluted canal. Ecotoxicology 12: 405-416.

Kochzius M. 1997. Length-weight relationships of fishes from a seagrass meadow in Negros Oriental, Philippines. Naga, 2 (3-4): 64 - 65.

Konan KF, Ouattara A, Ouattara M, Gourêne G. 2007. Weight-length relationship of 57 fish species of the coastal rivers in South-Eastern of Ivory Coast. Croatian Journal of Fisheries, 65(2), 4960.

Kulbicki M, Guillemot N and Amand M. 2005. A general approach to length-weight relationships for New Caledonian lagoon fishes. Cybium 29: 235-252.

LaLèyè PA. 2006. Length-weight and lengthlength relationships of fishes from the Ouémé River in Bénin (West Africa). Journal of Applied Ichthyology 22 (4) : 330-333.

Laurence B, Safiatou C. 2019. Relation Longueur-Poids Pour 15 Espèces de Poissons Exploitées dans la Lagune Ebrié, Côte d'Ivoire (Afrique de
l'Ouest). European Scientific Journal.Vol.15, No. 21 ISSN 1857- 7431.

Lauzanne, L. 1988. Les habitudes alimentaires des poissons d'eau deuce Africains. pp. 221-242. In : C. Lévêque, M. Bruton \& G. Ssentongo (ed.) Biologie et Ecologic des Poissons d'Eau Deuce Africains - Biology and Ecology of African Freshwater Fishes, ORSTOM, Collection Travaux et Documents 216, Paris.

Le Cren ED. 1951. The length-weight relationships and seasonal cycle in gonad weight and condition in perch (Perca fluviatilis). Journal of Animal Ecology, 20 (2): 201 - 219.

Lederoun D, Lalèyè P, Vreven E, Vandewalle P. 2012. Length-weight and length-length relationships and condition factors for 30 actinopterygian fish from the Mono basin (Benin and Togo, West Africa). Cybium 40 (4) : 267-274.

Legendre, M., \& Ecoutin, J.M., 1996. Aspects de la stratégie de reproduction de Sarotherodon melanotheron: comparaison entre une population naturelle (lagune Ebrié, Côte d'Ivoire) et différentes populations d'élevage.

Lelek A. et El Zarka S. 1973. Ecological comparison of the préimpoundments and post impoundments fish faunas of the river Niger and Kainji lake, Nigeria. Am. Geoph. Union, 17: 655-660.

Lewis DSC. 1974. The food and feeding habits of Hydrocynus forskalii Cuvier and Hydrocynus brevis Günther in lake Kainji, Nigeria. J. Fish. Biol., 6 (4): 349-363.

Lorenzen K. 2000. Population dynamics and management. Pp. 163-225. In: M. C. M. Beveridge, B. J. McAndrew, éds, Tilapias: Biology and Exploitation. Kluwer Academic Publishers, Great Britain, Fish and Fisheries series 25.

Lowe-Mc Connell RH. 1966. Man-made Zakes. Academic Press, London and New-York, 218 p.

Lowe-Mc Connell RH. 1975. Fish communities in tropical freshwaters: their distribution, ecology, and evolution. Longman, London, $337 \mathrm{p}$.

Mikembi A, Mamonekene V, Tenda H, Ngot F, Vouidibio J. 2019. Relations longueurs-poids et coefficients de condition pour 13 espèces de poissons de la rivière Dzoumouna, affluent du cours inférieur du fleuve Congo (République du Congo). Journal of Animal and Plant Sciences, 39 (1), 6384-6393.

Mahomoud WF, Amin AMM, Elboray KF, Ramadhan AM, El-Halfawy M. 2011. Reproductive biology 
and some observation on the age, growth, and management of Tilapia zillii (Gerv, 1848) from Lake Timsah, Egypt. International Journal. 3 :15-25.

Mano K. 2016. Fish Assemblages and Aquatic Ecological Integrity in Burkina Faso PhD thesis, University of Natural Resources and Life Sciences, Vienna Austria, p. 283.

Montchowui E, Kogbeto MJ, Laleye P. 2009. Weightlength relationships for commercial fish species caught in Lake Hlan in Benin (West Africa). International Journal of Biological and Chemical Sciences, 3 (3).

Moutopoulos DK and Stergiou KI. 2002. Length-weight and length-length relationships of fish species from the Aegean Sea (Greece). Journal of Applied Ichthyology 18: 200 - 203.

MRAH 2018. Rapport d'activités post CASEM, Burkina Faso.

Muzzalifah AH, Mashhor M, Siti AMN. 2015. Lengthweight Relationship and Condition Factor of Fish Populations in Temengor Reservoir: Indication of Environmental Health. Sains Malaysiana 44 (1) : 61-66.

Mukankomeje R, Laviolette F, Descy JP. 1994. Régime alimentaire de Tilapia, Oreochromis niloticus, du lac Muhazi (Rwanda). In Annales de Limnologie-International Journal of Limnology (Vol. 30, No. 4, pp. 297-312). EDP Sciences.

Nehemia A, Maganira JD, Rumisha C. 2012. LengthWeight relationship and condition factor of tilapia species grown in marine and freshwater ponds. Agriculture and Biology Journal of North America, 3 (3), 117-124.

Njiru M, Okeyo-Owuor JB, Muchiri M, Cowx IG. 2004. Shifts in the food of Nile tilapia, Oreochromis niloticus (L.) in Lake Victoria, Kenya. African Journal of Ecology, 42(3), 163-170.

Obeng LE (ed). 1969. Man-made Zakes: the Accra symposium. Ghana Universities Press, Accra; $398 \mathrm{p}$.

Odat N. 2003. Length-weight relationship of fishes from coral reefs along the coastline of Jordan (Golf of Aqaba). Naga, 26 (1): 9-10.

Olurin KB, Aderibigbe OA. 2006. Length-weight relationship and condition factor of pond reared juvenile Oreochromis niloticus. World journal of Zoology, 1 (2), 82-85.

Oni SK, Olayemi JY, Adegboye JD. 1983. Comparative physiology of three ecologically distinct freshwater fishes, Alestes nurse Ruppell, Synodontis schall Broch \& Schneider and Tilapia zillii Gervais. Journal of Fish Biology, 22(1), 105-109.

Ouéda A, Guenda W, Ouattara A, Gourène G, Hugueny B, Kabré GB. 2008: Seasonal diet shift of the most important fish species in a sahelosoudanian reservoir (Burkina Faso). Journal of Fisheries and Aquatic Science 3 (4): 240-251.

Ouédraogo R, Soara A, Zerbo H. 2015. Caractérisation du peuplement piscicole du réservoir de Boalin, Ziniaré (Burkina Faso) deux décennies après l'introduction de Heterotis niloticus. Int. J. Biol. Chem. Sci., 9(5): 2488-2499. DOI: http://dx.doi.org /10.4314/ijbcs. v9i5.20.

Paugy D, Fermon Y, Abban KE, Diop ME, Traoré K. 1999. Onchocerciasis Control Programme in West Africa: a 20-year monitoring of fish assemblages. Aquat. Living Resour. 12 (6) : 363-378.

Paugy D, Lévêque C, Teugels GG. 2003. Poissons d'Eaux Douces et Saumâtres de l'Afrique de l'Ouest. IRD: Paris, France.

Pauly D. 1983. Some simple methods for the assessment of tropical fish stocks. FAO Fisheries Technical paper, (234), FAO, Rome, Italy, $52 \mathrm{pp}$.

Pauly D, Moreau J. 1997. Méthodes pour l'Evaluation des Ressources Halieutiques. CEPADUES : Toulouse, collection polytech de l'INP de Toulouse.

Piquemal D. 1991. Inventaire et bilan des retenues d'eau au Burkina Faso. Les cahiers d'outre-mer, 44 (175), 259-280.

Poda JN, Traoré A, Sondo BK. 2004. L'endémie bilharzienne au Burkina Faso. Bull Soc Pathol Exot, 97 (1), 47-52.

Présidence du Faso. 2014: DECRET N²014791/PRES/PM/MRAH/MEF du 16 septembre 2014 portant adoption de la Stratégie Nationale de Développement Durable de la Pêche et de l'Aquaculture au Burkina Faso (SNDDPA). JO N47 DE 2014

Sidibé A. 2003. Les ressources halieutiques démersales côtières de la Guinée : exploitation, biologie et dynamique des principales espèces de la communauté à Sciaenidés. Thèse de l'ENSAR mention Halieutique, Agrocampus Rennes, 320 p.

Sirima O, Toguyeni A, Kaboré-Zoungrana C. 2009. Faune piscicole du bassin de la Comoé et 
paramètres de croissance de quelques espèces d'intérêt économique. Int. J. Biol. Chem. Sci., 3 (1): 95-106. http://dx.doi.org/10.4314/ijbcs.v3i1.4274 0.

Stergiou KI, Moutopoulos DK. 2001. A review of lengthweight relationships of fishes from Greek marine waters. Naga 24 (1 \& 2), 23-39.

Thomas J, Venu S, Kurup BM. 2003. Lengthweight relationship of some deep-sea fish inhabiting the continental slope beyond $250 \mathrm{~m}$ along the west coast of India. Naga, 26(2) : 17-21.
Yonkeu S, Traore O. 2009. Etude d'impact environnemental et social de la mise en place du barrage a buts multiples de Samandeni au Burkina Faso. Volet centrale hydroélectrique. Institut International d'ingénierie de l'eau et de l'environnement. 2iE Groupe EIER/ETSHER, Burkina Faso.

Vos LD. 1995. A systematic revision of the African Schilbeidae (Teleostei, Siluriformes) with an annotated bibliography. Annales-Musee Royal de l'Afrique Centrale. Sciences Zoologiques (Belgium).

\section{ANNEXE}

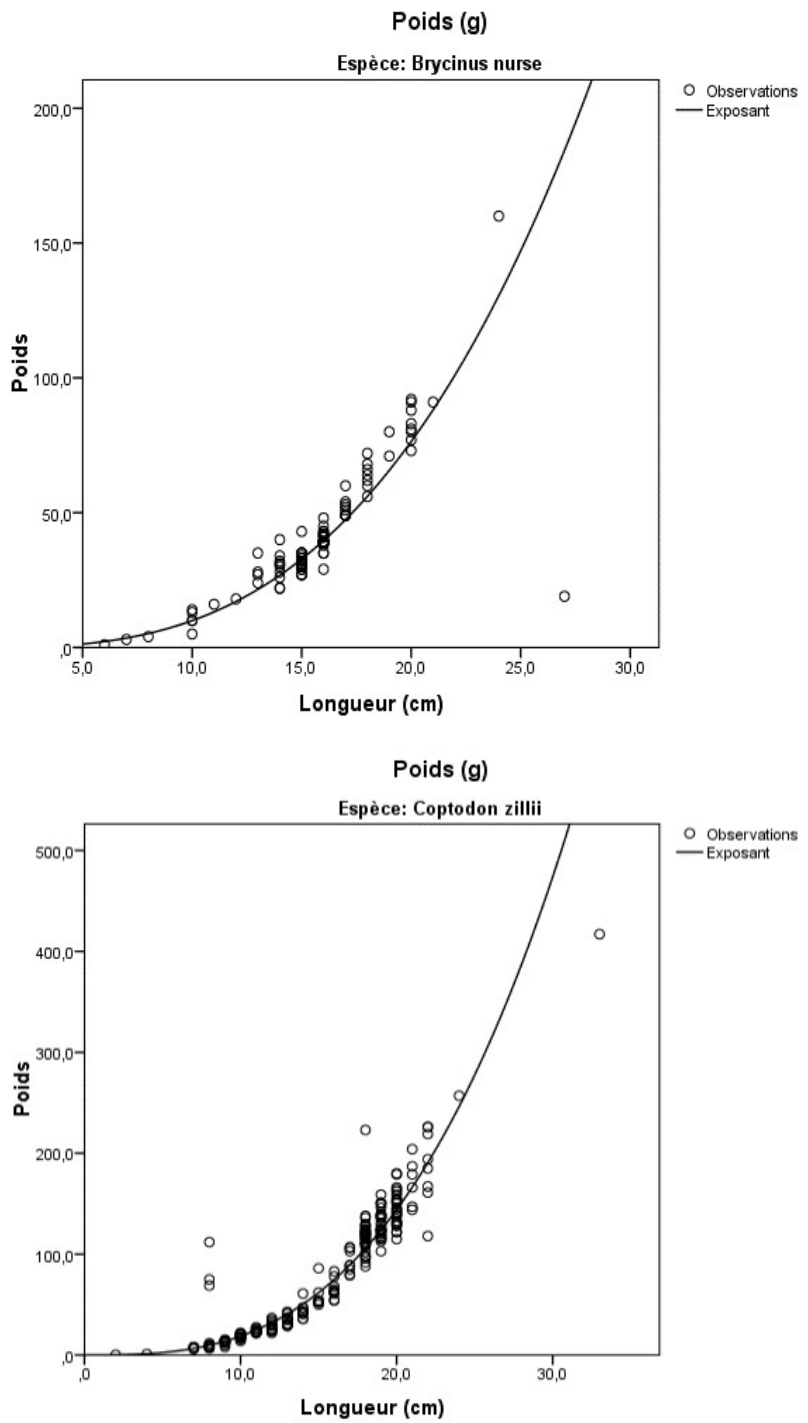


Minoungou et al., J. Appl. Biosci. 2020 Relation longueur-poids et facteur de condition de sept espèces de poisson du réservoir de Samandeni avant son ouverture à la pêche (Burkina Faso).
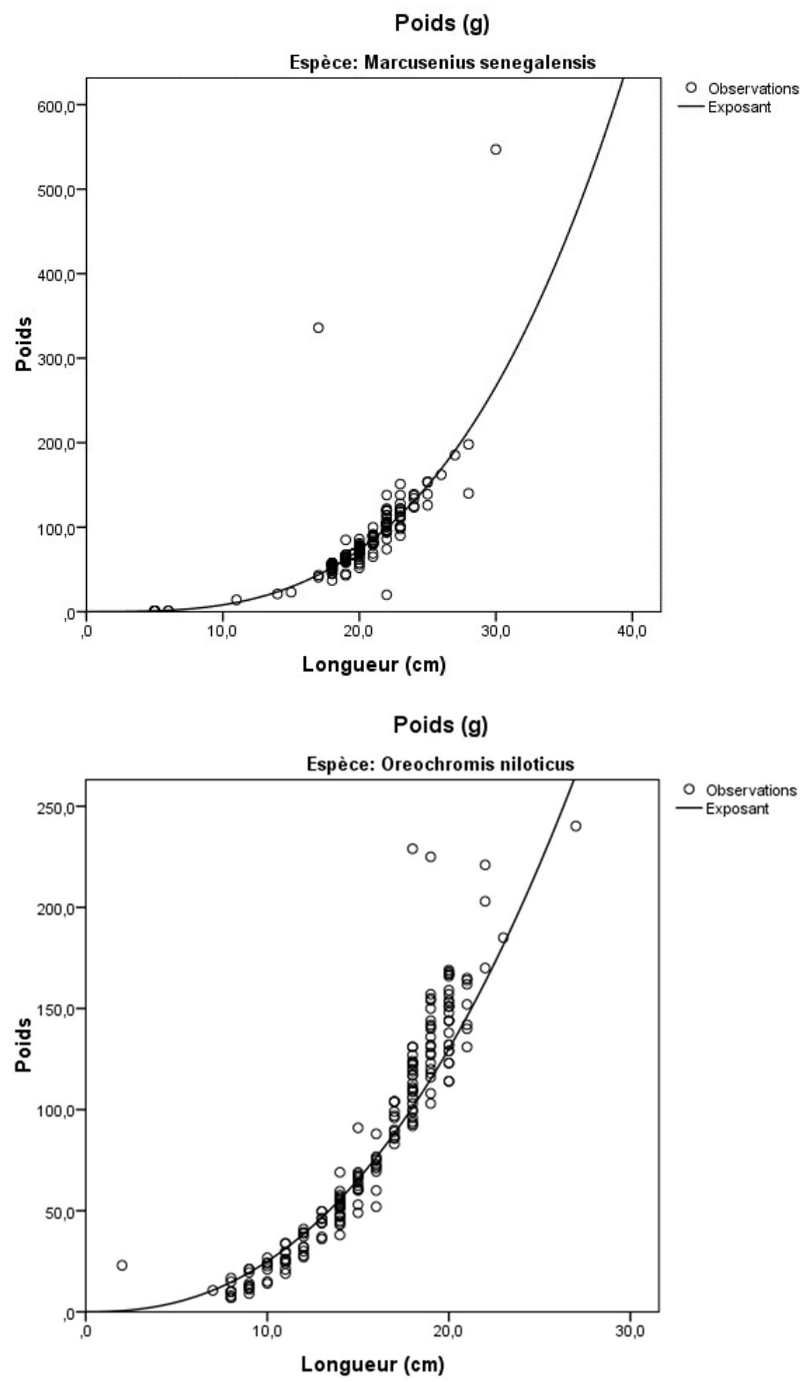

O Observations

Exposant 
Minoungou et al., J. Appl. Biosci. 2020 Relation longueur-poids et facteur de condition de sept espèces de poisson du réservoir de Samandeni avant son ouverture à la pêche (Burkina Faso).

Poids (g)

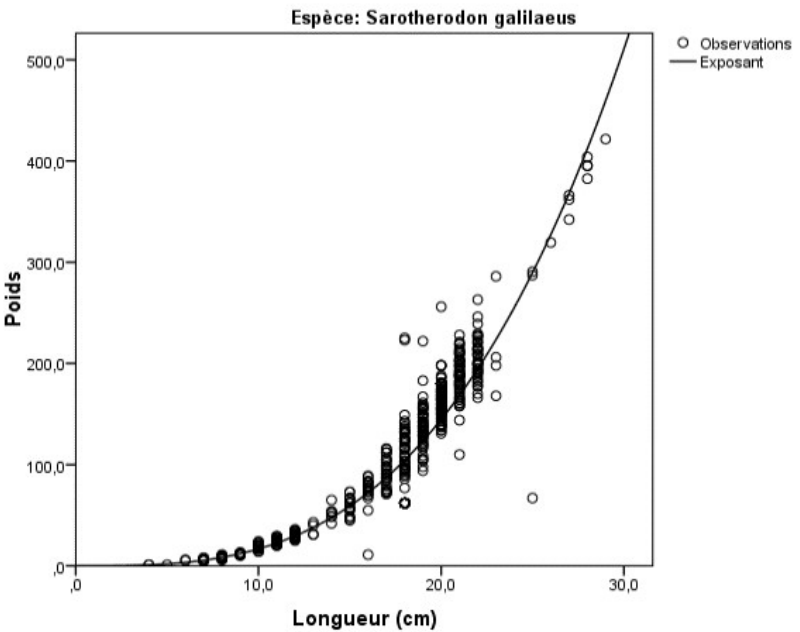

Poids (g)

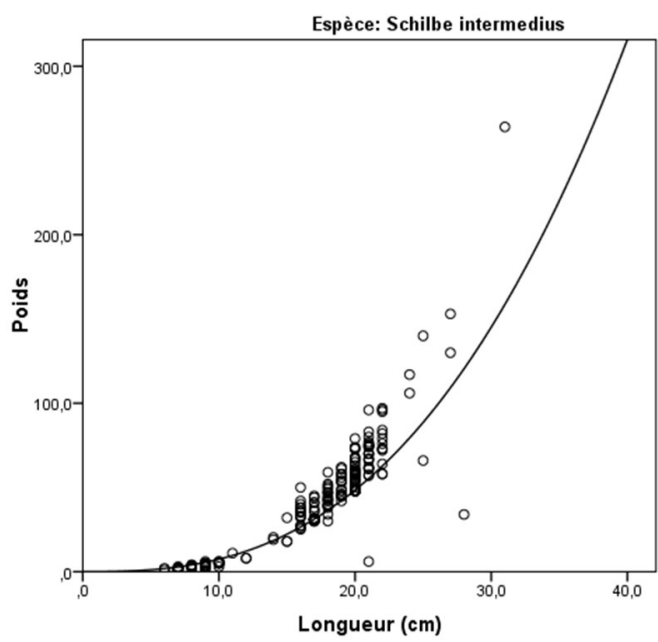

O Observations Exposant

Poids (g)

Espèce: Synodontis schall

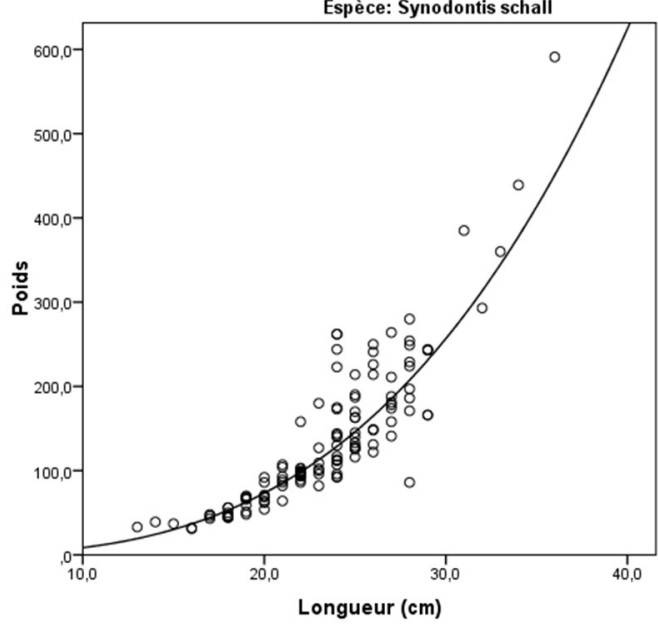

O Observations -Exposant 